\title{
«Articulación de la Educación Media con la Educación Superior en el Distrito Capital. Experiencia UNIMINUTO»
}

\author{
«Media and high school education articulation in Bogotá. Experience \\ UNIMINUTO» \\ «Articulação da Educação Média com a Educação Superior no Distrito Capital. \\ Experiência UNIMINUTO»
}

Por: Rafael Eduardo Parada Mendoza y Bejamín Barón Velandia (2015),

Bogota: Corporación Universitaria Minuto de Dios.

\author{
Daniela Medina Romero ${ }^{1}$ \\ Corporación Universitaria Minuto de Dios, Bogotá \\ Ingrith Yecenia Medina Martínez ${ }^{2}$ \\ Corporación Universitaria Minuto de Dios, Bogotá \\ ingrithmedina1993@gmail.com
}

1 Estudiante de la Licenciatura en Pedagogía Infantil e integrante del semillero de investigación: "¿Practicas para docente o prácticas para la vida?", Facultad de Educación de la Corporación Universitaria Minuto de Dios - UNIMINUTO.

2 Estudiante de la Licenciatura en Pedagogía Infantil e integrante del semillero de investigación: “¿Practicas para docente o prácticas para la vida?”, Facultad de Educación de la Corporación Universitaria Minuto de Dios - UNIMINUTO. 
Los cuatro capítulos presentados en el libro: Articulación de la Educación Media con la Educación Superior en el Distrito Capital (2015), dan muestra de un proceso investigativo. En el CAPítulo ı: Impacto de la articulación en la Educación Media, los autores exponen las consideraciones metodológicas y conceptuales tenidas en cuenta para la medición del impacto de la articulación en la Educación Media, junto a los análisis de los resultados obtenidos en dicha medición.

Del mismo modo, describen cómo los grupos focales con una mirada crítica, introspectiva, retrospectiva y ubicados en cada uno de los colegios del Distrito Capital, intervienen en planes de mejoramiento directamente en los grados de décimo y undécimo, con el propósito de que éstos obtengan los contenidos, las estrategias y las metodologías para el acceso y la permanencia en la Educación Superior.

Por consiguiente, se plantean dos estrategias para superar las carencias entre la Educación Media y la Educación Superior:

1. Aumentar las posibilidades de acceso a la educación técnica y tecnológica.

2. Reorientar la Educación Media hacia una formación para el medio laboral e integrada a la Educación Superior.

Las estrategias mencionadas son consideradas como medios que facilitan la construcción de una cultura para el trabajo y para la productividad, es decir, influyen en la reflexión de las condiciones vocacionales de cada estudiante y en la gestión de convenios o asociaciones con entidades oficiales y privadas.

Por tanto, para evaluar estos procesos se crearon e implementaron instrumentos que valoran las necesidades de los diversos contextos de manera crítica, esto es, de acuerdo al Proyecto Educativo Institucional — mencionado de ahora en adelante como PEl- , y, posteriormente, se lleva a cabo la potencialización de las capacidades de los alumnos. Estos instrumentos abarcan a todos los miembros de la comunidad educativa — directivas, docentes, estudiantes y padres de familia-.

El proceso evaluativo y el transcurso de la implementación de la articulación en los colegios del Distrito fue arduo, tanto para los estudiantes como para los docentes, debido, en principio, a las dificultades para establecer horarios con los estudiantes para determinar temas y contenidos con los pares académicos.

De otra parte, hubo dificultades en el desarrollo del Proyecto Curricular Articulado en el que están presentes los dos currículos: el currículo de cada colegio y el currículo de la Corporación Universitaria Minuto de Dios - UNIMINUTO; no obstante, se concluyó el proyecto con el apoyo y el compromiso de ambas partes 
en lo que respecta a los gastos de la articulación. Además, los docentes, debido a su interés por capacitarse, respondieron satisfactoriamente a las necesidades de los estudiantes y, a su vez, al nivel de exigencia requerido por la institución. De la misma forma, los estudiantes se apropiaron de las posibilidades para dar continuidad a sus estudios, mientras que los padres de familia garantizaron su apoyo y su participación en estos escenarios formativos.

En cuanto al CAPítulo ॥: Política educativa, este presenta las políticas públicas y educativas dentro del periodo comprendido entre 1998 y 2013, muestra las generalidades y el panorama de la política educativa colombiana; asimismo, relaciona la estadística de cobertura neta en la educación secundaria y media en el Distrito Capital.

Allí se define la política pública como un conjunto de relaciones interdependientes, adoptadas por un grupo de personas con intereses sociales, las cuales se enseñan y se explican a cada uno de los ciudadanos. Se hace una reflexión sobre el impacto y la acción social que interfieren en temas económicos y democráticos del país.

Por otro lado, se expone el conocimiento cultural e histórico de Colombia, manifestando que éste cambia en la medida que la concepción de educación se convierte en un bien económico y se transforma en términos de bienes y servicios; así, los maestros se reconocen como funcionarios del desarrollo de mercado, los rectores como gerentes institucionales y las escuelas como lugares de gestión. En consecuencia, la descripción de los acontecimientos evolutivos relevantes del medio educativo se escenifican en las últimas tres administraciones:

- Ley 30 de 1992: se refiere a las pautas para la contratación docente y el mejoramiento de la calidad educativa. Muestra los principios y la apuesta que realiza el sistema educativo y todo lo referente al control fiscal.

- Ley 115 de 1994: afirma el replanteamiento de las estrategias pedagógicas en función de concientizar al ciudadano --estudiante- de su responsabilidad política y su necesario aporte para el desarrollo del país. Trata la estandarización de la educación y el surgimiento de los Proyectos de Aprendizaje.

- Periodo entre 1994 y 1998: plantea la necesidad de mejorar el bienestar de los maestros, la importancia de formar profesionales en el exterior y la creación de doctorados en las universidades. Además, cita el Decreto 3012 que hace referencia a la organización y funcionamiento de las escuelas normales superiores.

- Periodo entre 1998 y 2002: aparece el proceso evaluativo para los maestros como condición de permanencia en el cargo y, en 
la misma medida, para llevar a cabo la actualización de prácticas docentes. Se exponen los Decretos 2277 y 1278, los cuales están asociados al escalafón.

Por último, con el empalme de las leyes y acuerdos pactados en los periodos mencionados, dentro del proceso de articulación, se considera la aprobación del grado doce, el cual tiene por objeto facilitar la continuidad de la Educación Media y la Educación Superior.

Respecto al CApítulo II: Enfoque de la investigación, se relaciona el enfoque praxeológico con el tipo de investigación que orientó al proceso investigativo, y los aportes de los instrumentos utilizados en el mismo; de igual manera, expone las características a observar, la población, la muestra de estudio y la perspectiva sobre el impacto de la articulación.

A partir de la investigación praxeológica, entendida como una intervención, se tiene por objeto que el investigador se involucre y adquiera un compromiso serio ante este ejercicio, además, pretende que el estudiante adquiera experiencias y comprenda el tema a investigar con mayor cercanía. De tal forma, para UNIMINUTO, en especial para la Facultad de Educación, la praxeología se entiende como un discurso reflexivo y crítico sobre la práctica y la acción sensata, en la cual se busca el mejoramiento de oportunidades, la coherencia y la eficiencia de los procesos investigativos. Esta metodología busca observar, analizar, reflexionar e interpretar el mundo para participar, intervenir y transformar la realidad en nuestro país.

Para ejemplificar y entender la idea del texto es necesario comprender la praxeología, junto al proceso de articulación de la Educación Media con la Educación Superior del Distrito Capital, de la siguiente manera:

- Ver: hace referencia a la lectura de la información arrojada por los instrumentos aplicados a los diferentes actores, con el fin de medir el impacto en la gestión académica y administrativa.

- Juzgar: es el momento de la interpretación, el análisis y la lucidez sobre la información adquirida en los colegios participantes.

- Actuar: aplica para las acciones consecuentes a lo observado y comprendido dentro del plan estratégico de acción y sobre las propuestas de mejoramiento a las necesidades de cada institución.

- Devolución creativa: aquí se proponen las recomendaciones hechas a las instituciones educativas partícipes en los procesos de articulación. Muestra el propósito de fortalecimiento de la Educación Media y el éxito de la proyección de Educación Superior. 
Al mismo tiempo, la investigación tuvo un enfoque social, puesto que buscó el mejoramiento de la calidad de vida de muchos jóvenes, quienes tenían una concepción de sí mismos y una visión sobre su futuro diferente a la que adquirieron con la articulación. Esto se logró gracias a que los estudiantes se involucraron en la Educación Superior y cambiaron un poco la proyección de su realidad a largo plazo, es decir, mejoraron las expectativas que tenían sobre su proyecto de vida.

En el CAPítulo iv se concluye que el proyecto de investigación sobre la articulación de la Educación Media con la Educación Superior sirvió para crear nuevas estrategias pedagógicas, respondiendo a las necesidades de los estudiantes y las expectativas de padres, docentes, instituciones de Educación Media del Distrito Capital y la institución de educación superior en cuestión; igualmente, se compartieron experiencias entre pares educativos de los colegios y la universidad.

Por otro lado, en las instituciones de secundaria se previó lo necesario para el fortalecimiento de los procesos pedagógicos y académicos con el fin de disponer de todo lo requerido para la formación posgradual. Así mismo, se preparó a los estudiantes para asumir su vida por medio de la experimentación de la educación como algo significativo, lo que favorece su permanencia en el sistema educativo y al mismo tiempo que permite la construcción de su proyecto de vida.

Por consiguiente, todas aquellas instituciones que hicieron parte de estos convenios de asociación lograron ver la necesidad de crear nuevas oportunidades para la Educación Superior, por lo que se amplió la cobertura y se crearon nuevos métodos para el ingreso a la Educación Superior. Se modificaron los proyectos curriculares articulados, los cuales podrán ser evaluados, analizados y actualizados a medida que pasa el tiempo y llegan nuevas tecnologías.

Para UNIMINUTO no se trataba sólo de fortalecer académicamente a los estudiantes, sino también a la institución desde un ente pedagógico y administrativo, logrando enlaces académicos de la Educación Media con la Educación Superior.

Con esta experiencia, a los estudiantes se les garantiza el derecho a la educación de calidad, se mejora su nivel educativo y se responde a sus necesidades actuales; empero, para esto es necesario tener un modelo educativo flexible y viable como lo es el de la articulación.

Desde el Estado, la concepción de educación se convierte en un bien económico y comienza a definirse en términos de bienes y servicios, lo cual se contrapone a la idea de que el maestro es aquella persona que brinda sus habilidades y capacidades en beneficio de los demás, limita a aquellos que buscan la creación y administración de espacios donde lo vital es la reflexión, la conciencia y la sensibilidad de las 
relaciones sociales, y los sujeta a espacios limitados donde el único propósito es la dirección y adquisición de conocimientos aislados a la realidad e identidad cultural. Puesto que tal concepción propone que los maestros y directivos son agentes y funcionarios de un sistema de mercado, esto da lugar a la obligación y no a la pasión, al gusto de ser docente; así, el acto de enseñar se convierte en una competencia en la que el docente ganador es quien obtiene más productos a la venta, y en la que su objetivo es la adquisición de bienes propios o el desarrollo no propiamente cultural, sino económico.

Durante el proceso investigativo, no sólo se conoce sobre la implementación de la articulación de la Educación Media con la Educación Superior, sino que se evidencia la importancia del trabajo en equipo para lograr un objetivo claro. Aunque no es fácil, consideramos que las personas profesionales deben asumir retos y es necesario trabajar arduamente para alcanzar una meta. De este modo interviene la praxeología trabajada en la Corporación Universitaria Minuto de Dios - UNIMINUTO, entendiendo con mayor cercanía este ejercicio investigativo, al mismo tiempo que se aplica.

Esta investigación da una pauta de inicio para mejorar los proyectos de vida de la comunidad educativa en las diversas instituciones, dado que se evidenció la necesidad de una mayor preparación tanto de los docentes de la universidad y en los maestros de las instituciones, como de los estudiantes. Lo anterior se gesta con el propósito de que el proceso de articulación prevalezca y se implemente en todas las instituciones educativas. 


\section{Referencias}

Parada, R., y Barón, B. (2015). Articulación de la Educación Media con la Educación Superior en el Distrito Capital. Bogotá: Corporación Universitaria Minuto de Dios - UNIMINUTO.

Cómo citar: Medina, D., y Medina, I. (2017). «Articulación de la Educación Media con la Educación Superior en el Distrito Capital. Experiencia UNIMINUTO». Praxis Pedagógica, 20, 139-145 